\title{
Beobot 2.0: Autonomous Mobile Robot Localization and Navigation in Outdoor Pedestrian Environment
}

\author{
Chin-Kai Chang
}

\author{
Christian Siagian
}

Laurent Itti
We present Beobot 2.0 [1], an autonomous mobile robot designed to operate in unconstrained urban environments. The goal of the project is to create service robots that can be deployed for various tasks that require long range travel. Over the past two years, Beobot has successfully traversed various paths across the USC campus, demonstrating its robustness in recognizing and following different types of roads, avoiding obstacles such as pedestrians and service vehicles, and finding its way to the goal.

Beobot utilizes a sixteen core computing platform [2], and is equipped with sensors such as front-facing cameras, an Inertial Measurement Unit (IMU), two Laser Range Finders (LRF), and wheel encoders. Beobot represents its environment in a hierarchical way. It uses a topological map for global localization and a grid occupancy map for local navigation. By having separate and targeted maps for these tasks, the system achieves a representation that is both detailed and scalable to describe vast environments such as a university campus.

The navigation system consists of two sub-tasks: road recognition and obstacle avoidance. The system recognizes the road visually, by utilizing image contour segments to detect the vanishing point, indicating the direction of the road [3]. In addition, it also tracks the road lines to estimate the lateral position of the robot. The use of segments proves to be critical as the road recognition performs robustly despite the presence of occluding pedestrians as well as shadows. As for obstacle avoidance, the robot uses a planar LRF to populate the grid occupancy map. The system then generates a rigid path to the goal using $\mathrm{A}^{*}$, and refines it using the Elastic-Band Algorithm [4]. Furthermore, the system then computes the motor commands, accounting for robot shape and velocity, using the Dynamic Window Approach [5].

To localize in the global topological map, the system models two extensively studied human visual capabilities within its Monte-Carlo Localization framework [6]. One is extracting the gist of a scene [7], a holistic statistical signature of the image, to quickly classify the robot segment location. The second is detecting and identifying the salient regions in the scene to pin-point the robot position. The localization system is responsible for informing the robot

C.-K. Chang and C. Siagian are with Department of Computer Science, University of Southern California, Hedco Neuroscience Building Room 3641, 10 Watt Way, Los Angeles, California, 90089-2520, USA. \{chinkaic, siagian\}eusc.edu

L. Itti is with the Faculty of Computer Science, Psychology, and Neuroscience, Univesity of Southern California, Hedco Neuroscience Building - Room 30A, 3641 Watt Way, Los Angeles, California, 90089-2520, USA. itti@pollux.usc.edu

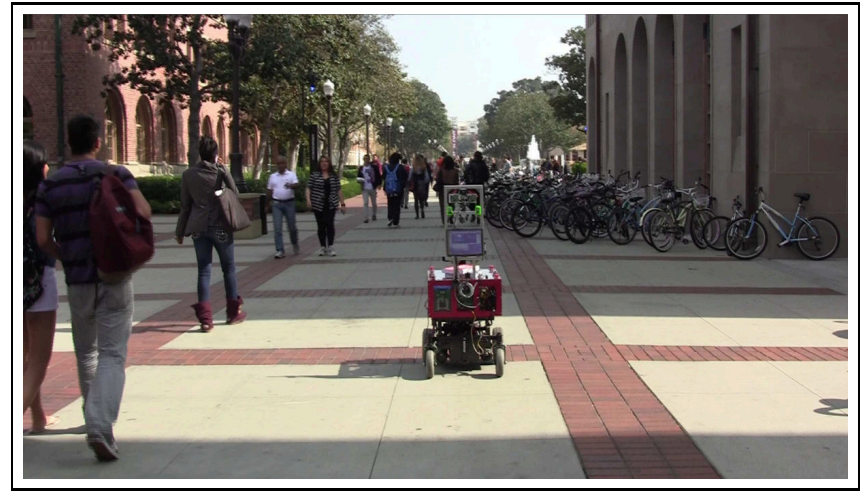

Fig. 1. Beobot 2.0 performs autonomous navigation and localization in a college campus, among pedestrians.

when and which way to turn at the intersection, and whether it has arrived at the goal location.

\section{ACKNOWLEDGMENTS}

The authors thank James Tanner for lending his voice to narrate the video. The authors gratefully acknowledge the support from the National Science Foundation (grant number CMMI- 1235539), the Army Research Office (W911NF11-1-0046), and U.S. Army (W81XWH-10-2-0076). The authors affirm that the views expressed herein are solely their own, and do not represent the views of the United States government or any agency thereof.

\section{REFERENCES}

[1] C. Siagian, C. K. Chang, and L. Itti, "Beobot 2.0," http://ilab.usc.edu/beobot2, December 2013, accessed: December $15,2012$.

[2] C. Siagian, C.-K. Chang, R. Voorhies, and L. Itti, "Beobot 2.0: Cluster architecture for mobile robotics," Journal of Field Robotics, vol. 28, no. 2, pp. 278-302, March/April 2011.

[3] C. Siagian, C. Chang, and L. Itti, "Mobile robot navigation system in outdoor pedestrian environment using vision-based road recognition," in Proc. IEEE International Conference on Robotics and Automation (ICRA), May 2013, bb, both first authors contributed equally.

[4] S. Quinlan and O. Khatib, "Elastic bands: Connecting path planning and control," in Proc. IEEE International Conference on Robotics and Automation (ICRA), 1993, pp. 802-807.

[5] D. Fox, W. Burgard, and S. Thrun, "The dynamic window approach to collision avoidance," IEEE Robotics \& Automation Magazine, vol. 4, no. 1 , pp. $23-33,1997$.

[6] C. Siagian and L. Itti, "Biologically inspired mobile robot vision localization," IEEE Transactions on Robotics, vol. 25, no. 4, pp. 861873, July 2009.

[7] — "Rapid biologically-inspired scene classification using features shared with visual attention," IEEE Transactions on Pattern Analysis and Machine Intelligence, vol. 29, no. 2, pp. 300-312, Feb 2007. 